\title{
Erratum to: Breast reconstruction using free medial circumflex femoral artery perforator flaps: intraoperative anatomic study and clinical results
}

\author{
Mai Shibuya $^{1} \cdot$ Toshihiko Satake $^{1} \cdot$ Reiko Nakasone $^{1} \cdot$ Marina Ogawa $^{1} \cdot$ \\ Mayu Muto ${ }^{1}$. Kazutaka Narui ${ }^{2} \cdot$ Kazunori Yasumura $^{1} \cdot$ Takashi Ishikawa $^{3}$. \\ Jiro Maegawa ${ }^{1,4}$
}

Published online: 28 November 2016

(C) The Japanese Breast Cancer Society 2016

\section{Erratum to: Breast Cancer \\ DOI 10.1007/s12282-016-0728-x}

Unfortunately, this article was published online with the incorrect title "Breast reconstruction using free medial circumflex artery perforator flaps: intraoperative anatomic study and clinical results". The correct title is given in this erratum.

The online version of the original article can be found under doi:10.1007/s12282-016-0728-x.

Mai Shibuya

maispe0719@yahoo.co.jp

1 Department of Plastic and Reconstructive Surgery,

Yokohama City University Medical Center, 4-57

Urafunecho, Minami-ku, Yokohama, Kanagawa 232-0024,

Japan

2 Department of Breast and Thyroid Surgery, Yokohama City University Medical Center, Yokohama, Japan

3 Department of Breast Oncology, Tokyo Medical University Hospital, Tokyo, Japan

4 Department of Plastic and Reconstructive Surgery,

Yokohama City University Hospital, Yokohama, Japan 